\title{
Agôn
}

Revue des arts de la scène

HS 2 | 2014

Mettre en scène le conte

\section{Conte et théâtre : quand le récit hante les dramaturgies jeunesse}

Le cas de Cendrillon de Joël Pommerat

\section{Marie Bernanoce}

\section{OpenEdition}

\section{Journals}

Édition électronique

URL : http://journals.openedition.org/agon/3109

DOI : 10.4000/agon.3109

ISSN : 1961-8581

Éditeur

Association Agôn

Référence électronique

Marie Bernanoce, "Conte et théâtre : quand le récit hante les dramaturgies jeunesse ", Agôn [En ligne], HS 2 | 2014, mis en ligne le 28 septembre 2014, consulté le 19 avril 2019. URL : http://

journals.openedition.org/agon/3109; DOI : 10.4000/agon.3109

Ce document a été généré automatiquement le 19 avril 2019

Association Agôn et les auteurs des articles 


\section{Conte et théâtre : quand le récit hante les dramaturgies jeunesse}

Le cas de Cendrillon de Joël Pommerat

\section{Marie Bernanoce}

1 Joël Pommerat est à ce jour l'auteur de trois pièces destinées à la jeunesse, toutes publiées dans la collection Heyoka jeunesse de chez Actes Sud-Papiers, Le Petit Chaperon rouge ${ }^{1}$ en 2005, Pinocchio ${ }^{2}$ en 2008 et Cendrillon ${ }^{3}$ en 2012, accompagnées d'un travail graphique caractéristique de la collection et qui fait de ces objets-livres l'équivalent «d'albumsthéâtre $»^{4}$.

2 Sur ces trois pièces, deux sont des réécritures de contes et, pour analyser Cendrillon, il m'a semblé important de replacer la pièce dans le cadre global des relations que les écritures dramatiques jeunesse entretiennent avec les contes. Cela nous amènera dans un premier temps à dresser un panorama des questions historiques et esthétiques qui se posent de façon générale. Puis, nous nous pencherons sur trois grands réseaux de réécritures de contes qui permettent d'éclairer la démarche de Joël Pommerat dans Cendrillon. Enfin, dans la dernière partie, nous tenterons de caractériser l'esthétique dramaturgique de la pièce dans ce qu'elle porte de spécifique en matière de rapport au récit et qui rejoint l'ensemble de la démarche de l'auteur, en tant qu'auteur- metteur en scène.

\section{Conte et théâtre : une histoire, un état des lieux}

3 La présence des contes dans l'écriture théâtrale moderne et contemporaine est difficile à quantifier, même si elle est indéniable, principalement dans la part du théâtre qui s'adresse explicitement aux jeunes. Dans son ouvrage de 2009, Le Théâtre jeune public : un nouveau répertoire, en appui sur un travail de recherche mené en thèse ${ }^{5}$, Nicolas Faure dressait le panorama suivant :

À partir de recherches effectuées sur la revue Théâtre en France, qui proposait aux compagnies souhaitant y figurer de faire connaître leurs productions, la création pour le jeune public de 1970 à 1990 s'appuierait, en moyenne, sur les sources suivantes (...) : 
- création collective signée par la compagnie : $9 \%$;

- création signée par le directeur : $22 \%$;

- création signée par une autre personne : $21 \%$;

- création non signée : $4 \%$;

soit au total : $56 \%$ de créations.

- adaptations (de contes, d'œuvres littéraires - de littérature générale ou d'enfance

et de jeunesse) : $24 \%$;

- textes dramatiques : $20 \%$, dont les deux tiers sont des pièces du théâtre général, souvent des classiques (de Goldoni à Beckett)...

- restent environ $8 \%$ pour les textes édités en direction du jeune public ${ }^{6}$.

Sur la base d'un corpus de cent dix-neuf textes publiés entre 1980 et 2002, Nicolas Faure complétait ainsi son constat, pour les personnages :

(total supérieur à 100 car les éléments se combinent)

- $50 \%$ des textes du corpus mettent en scène des personnages d'enfants ;

$-26 \%$ des personnages d'adolescents ;

- $80 \%$ des personnages d'adultes ;

- $30 \%$ des personnages merveilleux (animaux doués de parole, ogres, etc.).

Le merveilleux est donc loin de constituer une caractéristique du répertoire : seul un tiers des textes l'utilise ${ }^{7}$.

5 Pour ma part, lorsque j'ai élaboré le premier volume de mon entreprise de Répertoire critique du théâtre contemporain pour la jeunesse $e^{8}$, mon corpus comptait environ deux cents cinquante pièces. Il atteint aujourd'hui sept cents pièces, sur un total que j'estime désormais à plus de mille pièces publiées dans la sphère française et francophone (en français d'origine ou traduit) dans des collections spécialisées. Or, mon analyse nuance quelque peu les constats de Nicolas Faure.

Il est effectivement nécessaire, pour fixer les bases des relations entre le conte et le théâtre pour les jeunes, de rappeler que, dans les années 60-80, la part du conte a été fondatrice dans la construction de ce que je nomme le théâtre jeune public, c'est-à-dire ce qui est produit sur les scènes. Le théâtre destiné aux jeunes, à cette époque, était fréquemment synonyme d'adaptation et de réécriture de contes, souvent sous forme de création collective. Les bases esthétiques en étaient globalement celles d'un théâtre de distraction et/ou à connotation didactique, sans grande recherche novatrice, malgré des contre-exemples notables parmi lesquels il faut citer le travail de Catherine Dasté. Dans les années 80-90, la construction progressive d'un véritable répertoire de pièces de théâtre jeunesse a vu la part des adaptations de contes diminuer par rapport à ce qu'elle avait été les deux décennies précédentes. De véritables univers d'auteurs ont émergé dans l'adresse aux jeunes, jusqu'à construire des œuvres que l'on peut considérer aujourd'hui comme des classiques du domaine, et je m'emploie à le montrer dans mon entreprise de Répertoire critique. Or, ces auteurs jouent davantage sur l'originalité de leur propre créativité que sur la réécriture et l'adaptation en quelque sorte exogènes des contes. Bien sûr, cela n'exclut pas que des dramaturgies inventives s'appuient sur l'univers des contes, plus ou moins directement, dans des formes de détournement complexes, non "paresseuses ", pour reprendre la terminologie de Jean-Pierre Sarrazac ${ }^{9}$.

7 C'est en partant de ce constat que j'ai été amenée à proposer la distinction entre " adaptation récréation » et " adaptation-recréation ». Les « adaptations-récréations » se présentent comme des adaptations ludiques à visée patrimoniale, dans lesquelles la modernisation des contes tend souvent à la parodie. Les "adaptations-recréations " quant à elles développent des adaptations en forme de quête esthétique, où « la présence 
du conte est moins thématique qu'esthétique, devenant matière à recherche de théâtralité revivifiée $»^{10}$.

8 Cette dichotomie peut évidemment être pensée de façon plus complexe en termes de relation aux contes sources. Christiane Connan-Pintado propose ${ }^{11}$ les trois catégories suivantes, dans ce qu'elle nomme des «transpositions» du conte au théâtre: les « transpositions minimales ", les « transpositions parodiques » et « les transpositions réappropriations ». Les premières "portent généralement le même titre que les contes sources et se contentent de substituer au récit linéaire les marques scripturales du théâtre ${ }^{12}$ ». Les transpositions parodiques "sont les plus nombreuses car la parodie domine de façon écrasante le champ des réécritures de contes et caractérise, en particulier, les transpositions les moins légitimes, souvent publiées chez des éditeurs à vocation pédagogique ${ }^{13}$ ». La dernière catégorie correspond à ce que j'appelle les adaptations recréations. Mais finalement la première de ces trois catégories représente peu de pièces, et parmi elles celles que j'ai proposé d'appeler "auto-dramatisation ", «transposition théâtrale par un auteur d'une de ses propres œuvres, très souvent narrative ${ }^{14} »$.

Comme on s'en doute, le chiffrage précis de ces différentes sortes d'adaptations de contes est difficile et il faudrait aussi ajouter à cette difficulté la prise en compte des phénomènes d'intertextualité, assez importants en nombre, y compris récemment.

Quoiqu'il en soit, mes lectures de ce corpus me permettent d'avancer une autre donnée, en termes d'évolution des relations entre conte et théâtre. En effet, semble se confirmer en cette seconde décennie du XXI ${ }^{\mathrm{e}}$ siècle une nette résurgence des réécritures de contes, du plus récréatif au plus recréatif. Et ceci est particulièrement le cas des réécritures et adaptations scéniques du Petit Poucet, assez nombreuses et sur lesquelles on ne peut manquer de s'interroger en termes sociologiques.

11 On peut même voir des œuvres théâtrales récentes clairement destinées aux adultes et qui se jouent de la matière d'un ou de plusieurs contes. C'est le cas de Au bois ${ }^{15}$ de Claudine Galea, réécriture du conte du Petit Chaperon Rouge qui entremêle la matière du conte, traditionnellement perçue comme un objet du théâtre jeunesse, et le point de vue très assumé de l'adulte sur cette matière du conte, le tout étant assorti d'une dimension fortement métathéâtrale.

12 Il y a là, et sans doute faut-il y insister, les traces d'une historicité des contes. S'ils sont souvent ressentis de façon anhistorique, du fait de leur fonctionnement patrimonial, comme la source mythique et archétypale à laquelle l'imaginaire humain s'abreuverait sans début et sans fin, en réalité leur matière, dans son apparition et son évolution, raconte l'histoire des mentalités et donc aussi celle des formes esthétiques.

13 C'est ainsi que dans l'ouvrage qu'il a dirigé, Perrault en scène. Transpositions théâtrales de contes merveilleux, 1697-1800 ${ }^{16}$, Martial Poirson dresse un panorama historique des plus intéressants, surtout si on le met en perspective avec les esthétiques théâtrales actuelles.

14 L'expression « contes de fées » est apparue en 1698, tandis que Perrault avait publié en 1697 Les Histoires ou Contes du temps passé, plus connues sous le titre Les Contes de la mère l'oye. Il prenait ainsi rang dans la "Querelle de la fable », en s'engageant clairement du côté des modernes, défendant le conte comme une des voies de recréation littéraire permettant de supplanter l'influence des Anciens.

15 Et cette époque marque le début d'une longue histoire de mariage entre contes et théâtre : il y eut ainsi jusqu'en 1800 une trentaine d'adaptations dramatiques des contes 
présentés par Perrault, adaptations explicites et directes. L'ensemble du XVIII siècle a été marqué par un goût prononcé pour les contes populaires aux résonances politiques. Parmi eux, non sans lien aux contes sadiens et libertins, «Barbe Bleue » arrivait en tête (avec dix adaptations théâtrales de 1746 à 1795), suivi par "Le Petit Poucet " et "Cendrillon». Le XIX siècle, marqué par l'émergence de la littérature de jeunesse, continua dans la voie des adaptations théâtrales de contes, consacrant une seconde fois les contes de Perrault, mais il préféra les contes galants et mondains, et parmi eux tout particulièrement « Cendrillon » (plus de trente versions dramatiques) et « La Belle au bois dormant ».

Il est alors important de noter, d'un point de vue théâtral, comment tout au long des XVIII ${ }^{e}$ et $\mathrm{XIX}^{\mathrm{e}}$ siècles semblent s'être tissés des liens d'une part entre les dispositifs illusionnistes du conte merveilleux et, d'autre part, le développement des arts de la scène et l'élaboration progressive de la notion de mise en scène.

\section{Conte et théâtre : des regards sur le monde, des esthétiques. Un terreau pour Joël Pommerat}

17 Les dramatisations de contes qui se sont développées dans la seconde moitié du $\mathrm{xx}^{\mathrm{e}}$ siècle et en ce début de $\mathrm{xxI}^{\mathrm{e}}$ siècle me semblent précisément participer, pour les plus recréatives d'entre elles, à un renouvellement actuel des dramaturgies textuelles et scéniques. Par ailleurs, il n'est sans doute pas fortuit d'observer que les contes qu'affectionnait le théâtre du XVIII ${ }^{\mathrm{e}}$ siècle en ses temps de bouillonnements politiques et sociaux, "Barbe Bleue ", «Le Petit Poucet » et " Cendrillon », semblent actuellement fort présents sur le devant de la scène théâtrale, ce qui peut intéresser un regard sociologique et anthropologique porté sur notre époque ${ }^{17}$. C'est la raison pour laquelle j'ai choisi de privilégier ces trois " réseaux ${ }^{18}$ » de réécritures dramatiques des contes, même si d'autres auraient également mérité que l'on s'y arrête, et en particulier les réécritures du Petit Chaperon Rouge. Cela va nous fournir le terreau de comparaisons fertiles avec la Cendrillon de Joël Pommerat qui sera ainsi éclairée par d'autres angles d'approche.

\section{Le réseau «Barbe Bleue »}

En me penchant sur les réécritures de contes et les relations familiales en leur sein ${ }^{19}, \mathrm{j}^{\prime} \mathrm{ai}$ pu circonscrire un réseau « Barbe Bleue ».

En 1989, avec leur pièce Barbe-bleue ${ }^{20}$, Jean-Pierre Moreux et Patricia Giros réécrivaient le conte de Perrault en mettant en avant sa cruauté et ses références sexuelles, sur fond de métathéâtre. La petite salle interdite se révèle fonctionner ici comme une extraordinaire machinerie théâtrale, pleine de boutons et de manettes. La mère vend littéralement l'une de ses filles à Barbe-Bleue, qui plus est en abandonnant l'aînée au profit de la cadette, et s'installe ensuite en filigrane une situation triangulaire: lors du bal des noces, organisé sur le champ, c'est la mère qui danse avec Barbe-Bleue en cherchant à le séduire, et les allusions à la rivalité mère-fille sont plusieurs fois explicitées. La belle-mère dans la Cendrillon de Joël Pommerat se met elle aussi en rivalité avec ses filles sur la base d'une modernisation qui cherche à coller à l'époque. Dans la pièce de Jean-Pierre Moreux et Patricia Giros, les cadeaux de Barbe Bleue à sa nouvelle et toute jeune épouse ressemblent à des poupées Barbie et à leurs accessoires. Chez Pommerat, ce sont les téléphones 
portables des filles et le vocabulaire très familier. On le sent, ce type de réécriture parle du contemporain et parle contemporain en se mettant en appui sur l'imaginaire des contes.

A l'opposé de cette forme de modernisation, Bruno Castan, dont la matière des contes irrigue toute l'œuvre théâtrale, construit une relation entre parole des contes et parole contemporaine d'une nature très différente. Dans sa pièce Coup de ble ${ }^{21}$, publiée tout d'abord en 1988 puis rééditée en 2001, il entremêle parole du conte et parole du monde contemporain dans une dramaturgie métissée assez unique, mixant procédés théâtraux et cinématographiques dans une forme particulière de "théâtre dans le théâtre dans le cinéma ». De plus, la structuration temporelle s'appuie sur un jeu complexe d'avancée dans la rétroaction, à saveur métathéâtrale et sous l'égide du personnage du grand $\mathrm{K}$ dans lequel on n'a aucune peine à discerner Kantor ${ }^{22}$. Mais on notera que si Bruno Castan mêle parole des contes et parole contemporaine, c'est en refusant toute modernisation directe des contes. Le conte de Barbe Bleue apparait dans la pièce comme le résultat d'une sorte de retour à la source, et l'enchâssement du contemporain dans le conte se réalise par le moyen des voyages de Barbe Bleue qui croise de réels faits divers prélevés par l'auteur dans des journaux. Cela donne une langue elle aussi métissée, entre archaïsme et langage actuel.

Plus récemment, en 2010, Jean-Michel Rabeux s'est aventuré dans le théâtre pour les jeunes avec une autre adaptation du conte, La Barbe Bleue $e^{23}$. On y retrouve les caractéristiques de la modernisation, de nature fortement burlesque, et l'on relèvera que la mère y joue rôle du témoin/récitant, la pièce se terminant par un happy end à la façon de La Belle au bois dormant.

Dans tous ces exemples, nous avons vu émerger des questions esthétiques autour du principe de l'adaptation des contes qui éclairent l'analyse de la pièce Cendrillon de Pommerat.

23 Par ailleurs, au-delà des problèmes de société concernant ce que l'on nomme désormais les "prédateurs sexuels ", le conte de Barbe Bleue semble offrir une chambre d'échos aux questions concernant le rôle de la femme dans la société contemporaine et sans doute estce là une des raisons de la grande présence des adaptations de ce conte. Or, nous pouvons remarquer que cette thématique n'est pas étrangère à la Cendrillon de Pommerat.

\section{Le réseau « Petit Poucet »}

24 Ce réseau, quant à lui, est en plein développement cette dernière décennie. De 2001 à 2014, on peut ainsi répertorier une quinzaine de dramatisations directes du conte, d'En attendant le Petit Poucet de Philippe Dorin ${ }^{24}$ à Un caillou dans la botte de Simon Grangeat, pièce à ce jour inédite, en passant par Bled de Daniel Danis ${ }^{25}$, Mange ta main de Jean-Claude Grumberg $^{26}$, Petite Poucet de Claudine Galea ${ }^{27}$, Ah la la! quelle histoire de Catherine Anne ${ }^{28}$, Poucet pour les grands de Gilles Granouillet ${ }^{29}$, Le Petit Poucet de Caroline Baratoux ${ }^{30}$, Le Songe de Baya d'Eléonore His ${ }^{31}$, Poucet version XXIème de Marwil Huguet, Mon Petit Poucet de José Pliya, Le Petit Poucet ou du bienfait des balades en forêt dans l'éducation des enfants de Laurent Gutmann, ou encore, dans un titre aux connotations parodiques, Comment Marie ForteCuisse réussit à alléger le poids de l'histoire (et elle-même par la même occasion) de Nathalie Rafal. Si l'on prend en compte les figures symboliques dominantes que véhicule le conte, dont celle de l'ogre qu'il partage avec « Hansel et Gretel », les pièces relevant de ce réseau sont extrêmement nombreuses, sans compter les réécritures scéniques non publiées. Ce 
constat a ainsi nourri la conclusion de l'avant-propos de mon ouvrage Vers un théâtre contagieux:

Nombreuses sont les pièces qui réécrivent le conte (...) Nombreuses sont celles qui, plus indirectement, se donnent pour héros un enfant abandonné ou évoquent les cailloux blancs du conte. Nombreuses sont celles qui traitent de l'abandon, de l'exil, de l'émigration, de la solitude, de la perte de maison, de la mort du père ou de la mère, et de la quête ainsi générée. Ce qui pouvait être avant tout quête de formation pour grandir semble aujourd'hui prendre les contours d'une quête existentielle partagée par les adultes $^{32}$.

La grande résurgence de ce conte du Petit Poucet dans le théâtre pour les jeunes semble donc en lien avec la forte présence des thématiques de la solitude, de l'abandon, de la quête et de la peur de la dévoration. Or ces sujets, présents dans les pièces jeunesse, ne sont pas réservés au monde des enfants mais semblent bien concerner l'ensemble de la société : on y voit en effet beaucoup d'adultes en errance, et dont le chemin même semble perdu. C'est ainsi que la pièce de Carlos Liscano Ma famille traite de vente des enfants et des parents dans un monde marchand absurde et familier à la fois, ce que l'on retrouve dans Un caillou dans la botte de Simon Grangeat, où l'on peut envisager de louer des enfants.

27 C'est à la lumière de ces constats sur les thématiques véhiculées par les adaptations du «Petit Poucet» qu'il me semble intéressant d'analyser le personnage de la belle-mère dans la Cendrillon de Joël Pommerat. Sa quête absurde, forcenée et ostentatoire de reconnaissance sociale et personnelle en fait un personnage totalement perdu et déstructuré, comme le révèle nettement la fin de la pièce. Sans doute peut-on y percevoir quelques souvenirs de la mère coquette qu'est Arkadina dans La Mouette de Tchekhov. Cependant, nous sommes moins en face d'un personnage de nature psychologique ${ }^{33}$ que d'une figure emblématique de l'époque, correspondant aussi à des choix de jeu et donnant un propos nettement social à la pièce. La marâtre se fait alors emblème de toutes les violences des gens de pouvoir, tout du moins dans la sphère familiale, car on remarquera que la figure du Roi, à l'inverse, est traitée avec bienveillance et dotée d'une humanité compréhensive et mesurée. Pour ce personnage de la belle-mère, qui règne ostensiblement sur la dramaturgie de la pièce, on pourrait mettre Cendrillon en échos avec l'univers de Carlos Liscano dans Ma famille qui, rappelons-le, n'a pas été écrite initialement pour des jeunes, et l'on connaît la réticence de Joël Pommerat à accepter de rentrer dans la partition étanche entre théâtre généraliste et théâtre pour les jeunes.

Si, à l'inverse de cette lecture quelque peu pessimiste des thématiques du « Petit Poucet ", on se penche sur la manière dont Michel Serres analyse la Petite Poucette ${ }^{34}$ qu'il voit dans $^{\prime}$ la jeunesse contemporaine née dans les nouvelles technologies, en ces temps de troisième grande révolution qu'est notre époque, après les inventions de l'écriture et de l'imprimerie, le propos social se fait alors beaucoup plus optimiste. Michel Serres projette en effet sur l'image de l'homme contemporain qui perd sa tête, métaphoriquement tombée dans l'ordinateur, la légende de Saint-Denis, chrétien dont la tête, tranchée par un centurion romain, est devenue la condition du miracle puisque Saint-Denis poursuit son chemin pour monter vers Montmartre et être sanctifié. J'ai pu de même analyser ${ }^{35}$ comment la plupart des écritures théâtrales jeunesse, par la nature et les effets de l'adresse aux jeunes, construisent une relation à la joie, au sens que Clément Rosset donne à ce mot, qui relève de cette forme d'optimisme et sans doute de positionnement philosophique, au-delà de tous les désastres du $\mathrm{xx}^{\mathrm{e}}$ siècle. 
29 La pièce de Joël Pommerat Cendrillon s'inscrit dans ce terreau complexe des relations entre conte et théâtre contemporain pour les jeunes, entre joie et horreur. S'opposent ainsi la musique et les bruits. On est en effet frappé par la grande présence de la danse et de la musique, dans le texte et plus encore dans la mise en scène, comme des incarnations de la joie. A l'opposé, les bruits que font les oiseaux lorsqu'ils se fracassent sur les parois de verre de la maison de la belle-mère fonctionnent comme l'antithèse de la musique présente dans les fêtes organisées par le roi. On pourrait aussi analyser la danse de la scène finale entre le Prince et La très jeune fille, unis par un rythme joyeux endiablés ${ }^{36}$, comme l'image inversée de la marche contrainte de la belle-mère sur ses talons hauts: très sonorisés sur scène, ils deviennent une sorte d'anti-musique, un peu à la manière des talons de la bourgeoise dans le film de Jacques Tati, Mon oncle. Ils sont aussi l'absence de danse, la danse empêchée (la belle-mère ne peut pas danser à la première fête du roi et elle chute lamentablement). La musique et la danse sont du côté de la vie, figures de joie, le bruit des oiseaux et la marche contrainte du côté de la mort, figures d'impasse et de folie.

30 C'est sans doute au filtre de cette analyse, moins politique que poétique, qu'il faut lire et interpréter le personnage du Roi : il aime les fêtes et la musique comme il prône une harmonie humaniste, et l'on tient là les raisons ou les effets dramaturgiques des parallèles, commençant avec la nomination des personnages, entre les deux mondes du palais et de la maison. Sur scène, ces parallèles sont rendus sensibles du fait que les deux pères sont joués par le même comédien. Au centre de ces parallèles, il y a deux mères mortes, disparues dans le temps passé. Or, le père de la très jeune fille partage avec le roi, père du très jeune prince, un ancrage dans le temps présent de l'envie de vivre, de la joie. Le fonctionnement de ces deux pères vient alors se placer en contrepoint de la narratrice dont il permet sans doute de mieux comprendre le sens. Cette voix mystérieuse, faite de douleur et de difficulté à dire, vient «d'il y a très longtemps »: "Tellement longtemps que je ne me rappelle plus si dans cette histoire c'est de moi qu'il s'agit ou bien de quelqu'un d'autre $»^{37}$.

31 A cette voix du temps s'oppose la voix du présent, de l'hic et nunc que représentent les deux pères, et la voix de la narratrice, doublée par un homme qui fait des gestes presque graphiques (la figure paternelle au présent, la figure de l'auteur?), peut alors se lire comme la présence de la mère morte du prince.

32 La dramaturgie de la pièce est ainsi clairement construite autour de cette opposition entre passé et présent, mort et vie, rejoignant en cela la morale qui se dessine explicitement : la mort d'un être aimé, a fortiori la mère, ne doit pas ruiner le présent. Cette leçon de joie, formulée précisément par le roi, est aussi portée par la fée qui fonctionne comme un personnage médiateur entre espace de la maison et espace du palais. Dans la mise en scène, elle a les talons de la mère mais elle pousse la très jeune fille sur les marches du palais où se donnent des fêtes.

\section{Le réseau « Cendrillon »}

33 Les pièces jeunesse réécrivant le conte de Cendrillon sont beaucoup moins nombreuses que celles qui se nourrissent du Petit Poucet mais elles semblent actuellement en plein développement, la mise en scène de Pommerat contribuant à remettre ce conte sur le devant de la scène. Gageons que la multiplication actuelle des situations de divorce et de famille recomposée, autant de thématiques qui émergent dans le théâtre jeunesse depuis 
une dizaine d'années, amènera nombre d'auteurs et de metteurs en scène à repartir sur les traces de Cendrillon même si, dans ces situations sociétales contemporaines, la mère n'est pas morte.

\section{dans Vers un théâtre contagieux et dont voici la présentation synthétique :}

Dans une esthétique assez cinématographique, mâtinée de musique, La Fille aux oiseaux est constituée d'une succession de courtes scènes comme autant de tableaux, séparés par des noirs systématiques. Certaines des 27 scènes, toutes numérotées et titrées, ont pour personnages des animaux et surtout des oiseaux, très importants autant dans leur rôle de chœur que dans leur portée symbolique. Les oiseaux, à l'image de ceux du conte de Grimm, sont les auxiliaires ailés et maternels de Cendrillon, ceux qui, coûte que coûte, la rattachent au ciel dont sa marâtre lui refuse l'accès en la livrant aux cendres. Ce sont même les oiseaux qui ouvrent et referment la pièce : leur parole semble alors celle du temps, de l'histoire écrite, mais que colore une douce ironie tendant au burlesque ${ }^{42}$.

Pour ouvrir la comparaison entre les pièces de Bruno Castan et de Joël Pommerat, on notera la présence commune d'une esthétique musicale, rythmant très fortement la langue chez Castan et structurant avant tout la mise en scène chez Pommerat, même si ne sont pas exclus dans Cendrillon des effets de répétition prononcés. Mais là où Castan déploie une écriture baroque, Pommerat fait dans le minimalisme des structures langagières du quotidien.

On relèvera aussi le sort réservé aux oiseaux. Chez Castan, dans un univers fortement marqué par le merveilleux et où les animaux parlent, les oiseaux exercent le rôle d'adjuvants, dans le respect du conte de Grimm. Au contraire, Pommerat reprend le motif des oiseaux pour en proposer une image inversée, en négatif : les oiseaux sont en effet les 
révélateurs de la méchanceté morbide de la belle-mère, par le biais d'une scénographie de la transparence: les murs de la maison de la belle-mère sont constitués de verre transparent, dans une utopie moderniste qui est aussi massacre de la nature. On remarquera enfin que la relation aux cendres de l'âtre, très présente chez Castan, est largement gommée chez Pommerat: le personnage inspiré de Cendrillon, s'il donne le titre de la pièce, est nommé dans la liste de personnage La très jeune fille, et tel est le cas dans la nomination didascalique tout au long de la pièce. Pourtant, elle un prénom, Sandra, mais devient Cendrier dans la bouche de la Belle-mère et de ses deux filles (et aussi pour le père dont elle éteint les cigarettes addictives), et Cendrillon uniquement à la fin de la pièce, dans la bouche du prince, quand la pièce rejoint le conte plutôt que d'en partir, pourrait-on dire. A l'inverse, Bruno Castan joue le jeu des symboles bachelardiens opposant feu et ciel, terre et ciel.

41 Le point commun de ces deux pièces, du point de vue de la nomination du personnage principal, est cependant que dans les deux cas la jeune fille change de nom au fil de la pièce : dans La Fille aux oiseaux, elle est tour d'abord nommée par la voix didascalique Ma chérie, pour devenir ensuite Cendrillon, et redevenir Ma chérie sous l'effet de l'amour du prince, contaminant la nomination didascalique.

42 Autre point commun entre les pièces de Bruno Castan et de Joël Pommerat: la déconstruction de la traditionnelle clausule des contes, «Ils se marièrent et eurent beaucoup d'enfants ${ }^{43}$ ». Chez Castan, c'est la polyphonie burlesque, ad libitum, à laquelle se livre le chœur des oiseaux. Chez Pommerat, c'est le contraste construit entre l'image du jeune couple qui danse et le texte de la Narratrice, annonçant leur séparation future.

Mais il y a, pour finir, une grosse différence entre les deux pièces: Pommerat, en appui sur Perrault, gomme la cruauté du châtiment final raconté dans le conte de Grimm. Bruno Castan, à l'inverse, choisit de garder la force, disruptive et presque prométhéenne, du dénouement cruel du conte chez Grimm, châtiant les coupables. Par contre, Pommerat donne à voir à deux reprises la mort de la mère que la pièce de Bruno Castan la renvoie au hors scène, par la parole des oiseaux. On relèvera que dans l'entretien déjà mentionné Pommerat posait les choses en ces termes :

C'est la question de la mort qui m'a donné envie de raconter cette histoire, non pas pour effaroucher les enfants, mais parce que je trouvais que cet angle de vue éclairait les choses d'une nouvelle lumière. (...) C'est peut-être aussi parce que comme enfant j'aurais aimé qu'on me parle de la mort qu'aujourd'hui je trouve intéressant d'essayer d'en parler aux enfants.

Cette incursion dans les réécritures de contes par le théâtre jeunesse, on le voit, débouche sur la question des modes d'adresse aux jeunes. Le conte, par ses liens à la transmission orale, conduit assez naturellement à thématiser la parole et donc sa théâtralité. Nous avons déjà vu s'esquisser des pistes de lectures métathéâtrales, au-delà des palimpsestes. Dans la suite de cet article, nous essaierons d'explorer comment Joël Pommerat fait vivre la théâtralité de la parole et du récit dans Cendrillon, en référence à quelques autres écritures dramatiques jeunesse.

\section{La dramatisation de contes chez Joël Pommerat : des métamorphoses du récit}

La présence du conte dans l'ensemble du répertoire théâtral jeunesse, on a pu le mesurer, met en jeu des thématiques mais engage aussi fortement des positionnements 
esthétiques. Nous allons explorer ces liens, là où les sujets abordés ne comptent pas plus que la manière dont ils font théâtre, là où les thématiques du conte peuvent aussi émerger du fonctionnement dramaturgique. Dans un entretien avec Claudine Galea, intitulé « Ouvrir des puits », Joël Pommerat clôt sa réflexion sur ces mots : «Ce dont on parle, on ne le dit pas $»^{44}$.

\section{Théâtre pour la jeunesse, contes et épicisation} d'un conteur, Joël Pommerat s'inscrit clairement dans les mélanges entre l'épique et le dramatique, le récit et l'action, fréquemment explorés par les écritures théâtrales jeunesse et très souvent avec une très grande inventivité.

En effet, nombreuses sont les pièces jeunesse qui déconstruisent allègrement le dramatique aristotélicien. Rappelons la définition de l'épicisation, ou épisation, telle que l'analyse l'ouvrage Poétique du drame moderne et contemporain, Lexique d'une recherche dirigé par Jean-Pierre Sarrazac, dans le prolongement renouvelé des analyses de Peter Szondi, étudiant les effets de la romanisation du théâtre vécue par le $\mathrm{XIX}^{\mathrm{e}}$ siècle :

Epiciser le théâtre, ce n'est [donc] pas le transformer en épopée ou en roman, ni le rendre purement épique, mais y incorporer des éléments épiques au même degré qu'on $\mathrm{y}$ intègre traditionnellement des éléments dramatiques ou lyriques. L'épicisation (ou épisation, sur le modèle de l'allemand Episierung) implique donc le développement du récit sans être une simple narrativisation du drame. (...)

Le sujet épique met en jeu, en scène, une forme narrative dont les modalités peuvent tout autant se retrouver dans l'usage du récit - pour sa forme la plus simple - que dans celui du montage ou du fragment: dans tous les cas, le sujet épique introduit une rupture de l'action dramatique telle que l'a défini Aristote dans son principe d'unité, de continuité ou de causalité. La fiction se transforme alors en réflexion. ${ }^{45}$

Un très grand nombre de réécritures de contes relèvent de cette épicisation. Prenons l'exemple de cet autre réseau de contes que nous n'avons pas abordé, celui du «Petit Chaperon rouge » dans lequel s'est inscrit Joël Pommerat.

Une des pièces fortes de ce réseau est Le Petit Chaperon Uf de Jean-Claude Grumberg ${ }^{46}$. C'est l'histoire du «Petit Chaperon rouge » passée au tamis de l'histoire du $\mathrm{xx}^{\mathrm{e}}$ siècle et de l'holocauste. Le loup est un caporal du nom de Wolf, le loup en allemand. La liste de personnages le présente ainsi : Wolf, loup déguisé en caporal, parle français avec accent loup. Il signifie à la petite fille que comme enfant Uf, dont la mention est portée sur ses papiers, elle ne peut plus avoir droit à toutes sortes de choses, son capuchon rouge en premier qu'elle doit remplacer par un vieux capuchon jaune, tout miteux. Or la pièce bascule dans ce que j'ai analysé comme une « esquive des fins » dont Jean-Claude Grumberg explore, de pièce en pièce, les effets de connivence à la fois humoristiques et très sérieux, relevant de l'épicisation. En effet la petite fille décide d'arrêter de jouer :

PETIT CHAPERON

Non non je joue plus, je veux plus être le Petit Capuchon Uf ou Jaune, je veux être dans la vraie histoire.

WOLF

Tu es dans la vraie histoire. PETIT CHAPERON

Non non, dans la vraie histoire du Petit Chaperon Rouge, la jolie histoire, celle qui fait pas peur et que tout le monde comprend. C'est dans celle-là que je veux être. 
WOLF

Bon ben comment on fait alors?

PETIT CHAPERON

On arrête tout et je raconte aux petits enfants la véritable histoire du Petit

Chaperon Rouge sans Ufs ni Oufs.

WOLF

Bon ben raconte

Wolf s'installe, ôte son masque de loup et écoute. ${ }^{47}$

50 Cet exemple est emblématique d'une des tendances des jeux épique/dramatique à l'œuvre dans le répertoire jeunesse, qui participe de ce que j'ai analysé comme un «humour multicolore ${ }^{48}$, dans la suite de la réflexion de Mireille Losco-Lena dans "Rien n'est plus drôle que le malheur". Du comique et de la douleur dans les écritures dramatiques contemporaines ${ }^{49}$. Or, il me semble que l'on peut retrouver cette drôlerie du malheur dans le théâtre de Pommerat.

51 Sa réécriture du « Petit Chaperon rouge » en offre l'illustration, associée à un autre jeu épique, celui du dédoublement des personnages, ici selon ce que j'appelle le double théâtralisé. Se construit dans la pièce, ce qu'accentue la mise en scène, un intéressant jeu de dédoublements successifs que l'on pourrait analyser à la lumière des interprétations de Bettelheim: la mère se dédouble en bête monstrueuse et l'enfant, partant chez sa grand-mère, se trouve doublée d'une ombre qui ressemble à sa mère dans sa dualité :

Il n'y avait plus que son ombre à côté d'elle

son ombre

avec laquelle elle pourrait se sentir encore un peu en sécurité

Une ombre très belle qui ressemblait par chance un peu à sa maman..$^{50}$

Cette ombre joue avec elle un jeu normal en termes de réalisme: elle disparait quand l'enfant quitte le plein soleil. Mais, sur le plan symbolique, on a l'impression qu'elle ne veut pas totalement protéger sa fille : geste d'abandon, geste pour la laisser grandir? Et le loup qui apparaît alors dans le touffu de la forêt prend tout naturellement la place de l'ombre qui quitte la petite fille... Le récit se conclut sur un décrochage temporel qui l'inscrit dans notre présent :

Aujourd'hui la petite fille est devenue une grande femme comme sa maman et elle se souvient très bien de toute cette histoire.

Sa maman qui est vieille habite une maison qui n'est pas très loin, ce qui est plus pratique pour se voir souvent. ${ }^{51}$

On le voit, à la fois nous ne sommes pas loin du happy end des contes et, dans le même temps, nous y sentons un humour grinçant déplaçant les codes du conte, en tout cas ceux de Perrault. Cela permet de faire résonner mieux encore le détournement de ces codes que nous avions déjà perçu à la fin de Cendrillon.

Mais la présence des contes ne se limite pas à ces détournements de récit.

\section{La voix didascalique du conte}

55 Au-delà des réécritures de contes, on voit en effet à l'œuvre une épicisation plus globale des écritures théâtrales jeunesse dans la proximité du contage qui m'ont amenée à parler de voix didascalique du conte. J'ai pu en effet constater la forte présence, en marge de l'adresse habituellement abordée du point de vue du seul dialogue, de ce que j'ai défini comme une adresse didascalique : 
Celle-ci construit un lien direct avec le lecteur, pour une bonne part non loin des formes orales du conte. Ses manifestations explicites sont la présence du « on », du « nous », et même du « vous » et du « Je ».52 Marie-Claire Le Pavec, Mamie Ouate en Papoâsie (Actes Sud-Papiers, 1989), fondatrice du genre même si elle accompagnait alors tout un mouvement de fond. Voici le début de «L'état du lieu », dont elle constitue le long texte didascalique initial :

Blupblup. C'est le nom de l'île, une île minuscule. C'est assez loin d'ici.

Administrativement, l'île Blupblup dépend d'une île géante, la Papoâsie. Elle n'a plus qu'un habitant, Kadouma. Les autres, eh bien ils sont morts, ou ils sont partis, ou ils se sont effacés dans le paysage, c'est comme on voudra!

Comme tous les Blupblutiens, Kadouma est grand et noir de peau, il a l'âge que vous lui donnerez, il parle parfaitement notre langue, il ne roule pas les "r", on ne sait pas pourquoi, c'est comme ça! !53

«nous » dont il est le complément presque naturel:

5. Le rêve

La Papoâsie, avec sa demi-lune et quelques étoiles plein ciel, s'est faite belle comme un rêve. Et les cris des animaux la nuit s'accordent à merveille. Ce concert n'est troublé que par une fausse note, le ronflement permanent de Mamie Ouate sous la tente. Puis une grande ombre se déplace, à qui voulez-vous qu'elle soit ? $^{54}$

7. La chimère

La vie a repris son cours en Papoâsie. Les paniers à provision sont légion, et l'appareil photo est sur son pied, toujours prêt à fonctionner. Mais ce jour-là, nos deux amis sont à quatre pattes, une loupe à la main, fascinés par un papillon qu'ils observent avec la plus grande attention. ${ }^{55}$ (onte, du dialogue au texte didascalique, de nombreuses œuvres théatrales pour la jeunesse développent une dramaturgie de nature musicale, faite de rimes (y compris entre le texte dialogué et le texte didascalique), de refrains, de répétitions, ce que j’ai choisi de nommer ritualisation dramaturgique. L'un des univers emblématiques de ce type de voix didascalique du conte est celui de Mike Kenny, où les personnages sans cesse passent du récit à l'action et l'inverse, dans des jeux d'échos, de reprises et de variations parfois vertigineux. Et il faudrait aussi évoquer les pièces jeunesse de Jon Fosse et toute l'originalité de la série Le Manuscrit des chiens ${ }^{56}$ : tressage complexe de points de vue, entremêlement de récit et d'action, de récit et de paroles, longues mélopées phrastiques qui emportent le lecteur.

Bien sûr, on trouvera dans ce répertoire des contes théâtralisés pour la jeunesse des exemples notoires de refus ou de mise à distance de l'épicisation. Olivier Py en est l'emblème. Dans ses trois pièces inspirés des frères Grimm, La Jeune Fille, le diable et le moulin et L'Eau de la vie (deux pièces publiées à l'école des loisirs respectivement en 1995 et 1999) et La Vraie Fiancée, une troisième dramatisation de conte publiée dans la collection Heyoka jeunesse d'Actes Sud-Papiers en 2008, l'ancrage de ses adaptations, malgré des nuances entre elles, peut se lire comme une revendication de la forme théâtrale dramatique, en appui sur des références shakespeariennes.

Agôn, HS 2 | 2014 
61 Au-delà de cet exemple, on peut aussi affirmer que l'ensemble des écritures théâtrales pour la jeunesse a fait œuvre de résistance par rapport aux éclatements forcenés de la narrativité théâtrale, développés à satiété par le théâtre depuis l'après-guerre dans ses formes post-modernes. Si les procédés de l'épicisation sont fortement développés dans le théâtre jeunesse, cela ne va jamais jusqu'à la dissolution de la forme dramatique. L'hypothèse que j'ai émise est que le rapport au récit et au contage a construit, dans l'adresse aux enfants et aux jeunes, par le rapport à l'enfance et à la jeunesse, des formes de barrières esthétiques contre les vagues du postdramatique que l'on peut considérer comme destructrices, sans pour autant s'engluer dans des esthétiques paresseuses ${ }^{57}$. Les réflexions récentes de Jean-Pierre Sarrazac dans Poétique du drame moderne $e^{58}$, pour ce qui est de l'ensemble du théâtre, confortent mon hypothèse. On peut même en tirer la conclusion que le théâtre pour les jeunes a été aux avant-postes d'un mouvement qui touche actuellement l'ensemble du théâtre.

Quelles sont alors, dans ce contexte, les formes de l'épique développées par Joël Pommerat dans son théâtre pour les jeunes?

\section{Le conte chez Joël Pommerat, une voix du récit}

63 La forme privilégiée de mélange épique/dramatique chez Pommerat tient, comme nous l'avons vu, au principe du conteur: dans Le Petit Chaperon rouge, il se nomme "L'Homme qui raconte», dans Pinocchio c'est "Le présentateur » et dans Cendrillon, c'est « une narratrice dont on n'entend que la voix ». Dans les trois cas, la pièce s'ouvre et se ferme sur cette parole du récit. Dans Le Petit Chaperon rouge, le conteur prend en charge presque la moitié du texte publié (vingt pages de dialogue pour dix-sept pages de récit conté), ce qui pousserait presque ce texte du côté de l'écriture mimodramatique. Dans ces trois pièces, les contes sont portés par une voix très présente, et un relais presque naturel semble se créer, à la lecture, entre cette voix du conteur et le texte didascalique.

On perçoit là un geste de base mettant en jeu un dramatique enchâssé dans le récit, ce qui relève d'une épicisaton que l'on peut qualifier de linéaire si on la compare aux mélanges épiques complexes mis en œuvre par d'autres écritures jeunesse. En cela, Joël Pommerat s'inscrit dans la droite ligne de la dette que le théâtre pour les jeunes a contractée non seulement par rapport aux contes, mais par rapport au contage. On connaît son goût du récit, sur lequel il s'est expliqué en maintes occasions, ainsi dans son entretien avec Christian Longchamp pour le site Théâtre contemporain ${ }^{59}$ :

Tout d'abord, il faudrait s'entendre sur ce qu'on appelle un conte. Je ne le sais pas vraiment moi-même. Peut-être entend-on une histoire ou plutôt un récit (SPN), qui se donne comme authentique, réel et qui évidemment ne l'est pas, et qui se développe avec des termes relativement simples et épurés, des actions qui ne sont pas expliquées psychologiquement.

Dans l'ouvrage qu'il a rédigé avec Joëlle Gayot, Joël Pommerat, Troubles, on peut lire ceci sous sa plume :

Dans les contes, par exemple, il n'est question que de familles Mais, comme dans mes pièces, ce n'est pas la personne en elle-même qui est importante, c'est ce qu'elle $v a$ faire en rapport avec l'autre. Et ce n'est pas cet autre qui est important, c'est la relation, le récit (SPN) qui va naitre et exister entre eux..$^{60}$

La présence du conteur dans les trois pièces jeunesse de Joël Pommerat relève ainsi d'un attachement fort au récit en tant que geste fondateur de l'adresse théâtrale, là où théâtre 
et conte se rejoignent dans l'ensemble de son travail d'« auteur de spectacles » comme il se présente lui-même ${ }^{61}$.

\section{Le conte chez Pommerat : une narration troublée}

67 Pour Joël Pommerat, écrire un spectacle et, sans doute, publier son texte (alors qu'il défend une démarche scénocentriste, non littéraire), cela relève d'une forte volonté d'adresse, mâtinée de métathéâtralité. De ce point de vue, c'est incontestablement le «présentateur» de Pinocchio qui retient le plus l'attention, disant aussi fortement « vous » que « je »:

Mesdames messieurs, bonsoir je vous souhaite la bienvenue. L'histoire que je vais vous raconter ici ce soir est une histoire extraordinaire, une histoire plus extraordinaire que vos rêves, et pourtant une histoire vraie... Mais avant de commencer à vous la dire, il faut que je vous parle de moi. Quand j'étais enfant, j'étais aveugle. Je n'y voyais rien. Mes yeux n'ont appris à voir clair que très tard, bien après que mes jambes eurent appris à marcher. Lorsque j'étais aveugle donc et que je ne voyais rien, voilà ce que je voyais.

(Noir)

Voilà. Ce que je voyais autour de moi quand j'ouvrais les yeux c'était ça : le noir. Pas très gai n'est-ce pas? Du coup dans ma tête je voyais énormément de choses. Ce qu'il y avait à l'intérieur de ma tête par exemple c'était ça.

(Une tête masquée apparaît.) ${ }^{62}$

Et « apparaissent » ainsi d'autres têtes, et une « assemblée de mannequins », avant que ne finisse par émerger, nés de la tête du présentateur, les personnages de l'histoire de Pinocchio. On peut donc analyser la structure dramaturgique de la pièce comme relevant du monodrame. Enchâsser ainsi l'action dans le récit, cela revient à construire un point de vue dont le centre vif se trouve dans la figure du conteur.

Commune aux trois pièces jeunesse et, au-delà d'elles, à la dramaturgie de Pommerat, la mise en œuvre du conteur présente cependant des nuances d'une œuvre à l'autre et ce fonctionnement mérite d'être analysé de près dans Cendrillon, comme le recommandait Patrick Laudet dans un entretien avec L. Carré en mai 2013 pour justifier, parmi d'autres raisons, le choix de cette pièce par l'Inspection Générale dans le cadre de l'option théâtre de Terminale :

Les choix de traitement de la narratrice qui intervient régulièrement dans la pièce peuvent être divers et riches et invitent à se pencher sur cette figure du narrateurprésentateur omniprésente dans l'œuvre de Pommerat. ${ }^{63}$

On notera au passage les expressions de "narratrice » et de "narrateur-présentateur ", et l'on sait que le texte même de la pièce présente une "narratrice». En ce qui me concerne, pour des raisons didactiques et pédagogiques, je préconise d'éviter le recours au terme de narrateur et de lui préférer le terme neutre de récitant. Le terme et la notion de narrateur posent en effet question. On peut bien sûr y recourir si l'on entend par là ce que Benjamin nommait le Erzähler, que j'ai induit ici à dessein en utilisant le mot conteur, et nous sommes bien dans ce cas avec le théâtre de Pommerat, comme le laisse entendre Patrick Laudet. Mais tout récitant n'est pas un conteur. Les pièces que j'ai appelées adaptations-récréations de contes ne recourent pas à un conteur mais se répandent dans une fausse théâtralité d'apparat, dont se joue me semble-t-il avec une certaine ironie le début de Pinocchio (texte et surtout mise en scène). Par ailleurs, il est des présences de récitant qui ne relèvent pas de la référence au genre narratif, soit qu'elles s'inscrivent dans les traditions du récital de poésie (et l'on pense à Rebotier et à sa pièce Les Trois Jours de la 
queue $d u$ dragon $\left.^{64}\right)$, soit qu'elles mettent en œuvre un mélange épique/dramatique profondément métathéatral en forme de machine à jouer. Du coup, il me semble nécessaire de réserver le terme de récitant à un fonctionnement dramaturgique de base que l'on peut ensuite colorer en termes de références génériques, sans oublier la contextualisation historique et son ancrage dans l'histoire de l'esthétique théâtrale et dans l'histoire des mentalités. ${ }^{65}$

Alors qu'en est-il précisément $\mathrm{du}$ récitant dans Cendrillon? Les distinctions terminologiques que nous venons d'esquisser vont se révéler utiles pour rendre compte de fonctionnements plus subtils qu'il n'y parait à première vue. Comme dans les deux autres pièces pour les jeunes de Joël Pommerat, le récitant y apparaît en effet de nature narrative. Mais nous allons observer dans cette pièce plusieurs détournements des principes narratifs du conteur, démultipliés par rapport aux deux autres pièces.

Dans Le Petit Chaperon rouge, à une héroïne au féminin correspond un conteur homme. Dans Pinocchio, à un héros masculin correspond un conteur homme. Dans Cendrillon, la figure du récitant se dédouble, d'une part en opposant/réunissant sexe masculin et sexe féminin et, d'autre part, en opposant/réunissant gestes et parole. Rappelons la présentation des personnages :

Une narratrice dont on n'entend que la voix

Un homme qui fait des gestes pendant qu'elle parle ${ }^{66}$

Lorsque l'on observe les gestes de l'homme dans la mise en scène, on se rend très vite compte qu'il ne s'agit en aucun cas d'une quelconque illustration des propos de la voix de la «narratrice». Aucune visée réaliste dans cette entreprise. Voix et corps sont traités volontairement sur le mode du décalage, ce que renforce le fort accent étranger de la comédienne « narratrice » italienne, Marcella Carrara.

Par ailleurs, les propos qui sont tenus par ce récitant double contribuent eux aussi à creuser la figure de cette narratrice et de la narration elle-même :

Je vais vous raconter une histoire d'il y a très longtemps... Tellement longtemps que je ne me rappelle plus si dans cette histoire c'est de moi qu'il s'agit ou bien de quelqu'un d'autre. J'ai eu une vie très longue. J'ai habité dans des pays tellement lointains qu'un jour j'ai même oublié la langue que ma mère m'avait apprise.

Ma vie a été tellement longue et je suis devenue tellement âgée que mon corps est devenu aussi léger et transparent qu'une plume. Je peux encore parler mais uniquement avec des gestes. Si vous avez assez d'imagination, je sais que vous pourrez m'entendre. Et peut-être même me comprendre. ${ }^{67}$

Ces usages du récitant se jouent ainsi de façon hybride de plusieurs contraires qu'ils tentent de relier tout en les opposant, féminin/masculin, incarnation/désincarnation, paroles/gestes, présence/absence et, ultimement, vie/mort. Contre les codes du contage narratif classique, qui cherchent à créer chez l'auditeur une adhésion et une connivence de l'ordre du merveilleux ${ }^{68}$, la figure du récitant est ici fortement tirée de façon métalinguistique et métathéâtrale du côté du poétique et de la machine à jouer. Et si Joël Pommerat recourt au terme de "narratrice ", il n'est pas nécessaire de prendre cela pour argent comptant. On peut en effet y discerner, on l'aura compris, un positionnement ironique qui réclame du spectateur une connivence en alerte, profondément double, entre adhésion et recul, dramatique et épique, qui fonde son esthétique du récit.

76 A cela s'ajoute la construction d'échos entre la parole du récit et les contenus de l'action, déjà à l'œuvre dans ses deux autres pièces jeunesse mais qui semble se renforcer dans Cendrillon. On ne manquera pas de remarquer ainsi combien la figure féminine du récitant 
se trouve placée sur le même plan symbolique que la mère de La très jeune fille, et le texte didascalique y invite clairement : " paroles incompréhensibles », « inaudible ${ }^{69}$. On peut donc percevoir une sorte de relais entre la mère de la « narratrice », la « narratrice » ellemême et la mère de la très jeune fille, cette dernière prenant clairement son envol en sortant de cette chaîne, qui plus est par une parole forte, audible, associée à l'action. C'est tout le sens de la scène où elle révèle au Très jeune prince la mort de sa mère, "fort " note le texte didascalique ${ }^{70}$ :

\section{LA TRES JEUNE FILLE}

Tu penses pas des fois qu'on est en train de te raconter des histoires avec cette

histoire ? (...)

LE TRES JEUNE PRINCE

Tu aimerais ça moi que je te dise que ta mère est morte?

LA TRES JEUNE FILLE

Ben tu pourrais...Tu pourrais me le dire.... Pace que c'est la vérité, ma mère est morte et tu sais moi aussi faut que j'arrête je crois de me raconter des histoires, me raconter qu'elle va peut-être revenir un jour ma mère, si je pense à elle continuellement par exemple non ! Elle est morte et c'est comme ça !11

Se raconter des histoires, et le mot revient très souvent, ne mène qu'à l'impasse.

\section{En forme de conclusion}

Dans un entremêlement entre le récit et l'action qui caractérise Cendrillon, où la voix de la « narratrice » intervient sans cesse, la narration est interrogée dans ses risques, à l'image de la magie de la fée qui peut devenir menaçante. Cendrillon dit et met en jeu les limites de la parole individuelle et sociale, à l'image du téléphone portable intervenant dans l'action qui éloigne autant qu'il rapproche. De façon métaphorique, Cendrillon opère ainsi une sorte de déterritorialisation du conte et du contage dont on peut percevoir les enjeux philosophiques. Le rêve de transparence que représente la « maison de verre » de la bellemère peut se lire comme le rêve dangereux d'une transparence de la langue : les impacts mortels des oiseaux sur les parois de verre se survivent, leur réalité est devenue sons. La langue est têtue et peut être porteuse de mort.

Pièce d'une métamorphose réussie grâce aux mots, Cendrillon se révèle aussi comme une pièce sombre où les mots et la narration peuvent être constitués des cendres de l'erreur, de la perte et de la trahison.

\section{NOTES}

1. Pommerat Joël, Le Petit Chaperon rouge, Illustrations de Marjolaine Leray, Heyoka jeunesse Actes Sud-papiers, 2005. La pièce a été rééditée par Actes Sud en 2014, sans illustrations, dans la collection Babel.

2. Pommerat Joël, Pinocchio, Illustrations d'Olivier Besson, Heyoka jeunesse Actes Sud-Papiers, 2008. 
3. Pommerat Joël, Cendrillon, Illustrations de Roxane Lumeret, Heyoka jeunesse Actes Sudpapiers, 2012. La pièce a été rééditée par Actes Sud en 2013, sans illustrations, dans la collection Babel.

4. Voir à ce sujet mes articles suivants : "L'album-théâtre ? Un genre en cours de constitution ", in Massol Jean-François (sous la dir. de), Les Cahiers de Lire Ecrire à l'école, Texte et images dans l'album et la bande dessinée pour enfants, Grenoble, CRDP de Grenoble, 2007, p. 121-135 ; «L'albumthéâtre »: typologie et questions posées à sa lecture », in Connan-Pintado Christiane, Gaïotti Florence, Poulon Bernadette (textes réunis par), L'album contemporain pour la jeunesse: nouvelles formes, nouveaux lecteurs? Modernités, $\mathrm{N}^{\circ}$ 28, Bordeaux, Presses Universitaires de Bordeaux, 2008, p. 39-52.

5. Faure Nicolas, De « jeune public » à « tout public »: analyse du répertoire théâtral francophone pour la jeunesse, Thèse de doctorat en Arts du spectacle, dirigée par Jean-Pierre Ryngaert, Paris III Sorbonne Nouvelle, novembre 2004.

6. Faure Nicolas, Le Théâtre jeune public: un nouveau répertoire, Rennes, Presses Universitaires de Rennes, 2009, p. 22-23.

7. Ibid., p. 46.

8. A la découverte de cent et une pièces, Répertoire critique du théâtre contemporain pour la jeunesse, Montreuil, Editions Théâtrales/Grenoble, SCEREN-CRDP de Grenoble, 2006.

9. Sarrazac Jean-Pierre, La Parabole ou l'enfance du théâtre, Belval, Circé, 2002.

10. «Place et nature des adaptations de contes dans le répertoire de théâtre pour la jeunesse : questions posées aux esthétiques théâtrales contemporaines ", in "Le conte à l'épreuve de la scène contemporaine, $\mathrm{XX}-\mathrm{XXI}^{\mathrm{e}}$ siècles ", Revue d'Histoire du Théâtre $\mathrm{n}^{\circ} 1$ et 2 , Paris, 2012, p. 171-180. Consulter aussi «Quand le conte se fait théâtre » in La Barbe bleue / Mon petit Poucet, L'avant-scène théâtre, Paris, 2010, p. 60-63.

11. Connan-Pintado Christiane, "Des personnages de contes en quête de représentation ", in Brillant-Annequin Anick et Bernanoce Marie, Enseigner le théâtre contemporain, Scéren/CRDP Grenoble, 2009, p. 93-104. Voir aussi Lire les contes détournés à l'école à partir des Contes de Perrault de la GS au CM, Paris, Hatier, 2010.

12. « Des personnages de contes en quête de représentation », op. cit., p. 94.

13. Ibid., p. 95.

14. "L'adaptation dans le théâtre jeunesse : contre «l'esthétique du débarras » in Les Cahiers de Lire Ecrire à l'école, L'adaptation, Grenoble, CRDP de Grenoble, 2008, p. 209-229.

15. Galea Claudine, Au bois, Editions Espaces 34, 2014.

16. Poirson Martial (dir.), Perrault en scène. Transpositions théâtrales de contes merveilleux, 1697-1800, Espaces 34, 2009.

17. Il y aurait là matière à mener une recherche de fond à la fois quantitative et qualitative sur les contes arrivant en tête des adaptations au $\mathrm{XX}^{\mathrm{e}}$ et sur les deux premières décennies du XXI ${ }^{\mathrm{ème}}$ siècle.

18. J'emploie le terme de réseau, en appui sur son usage en didactique de la littérature, dans le sens de constellation d'œuvres réécrivant des contes source de façons extrêmement variées, dans les catégories génériques définies.

19. «Les réécritures de contes dans le théâtre contemporain pour les jeunes : quel regard sur les relations familiales ? » in d'Humières Catherine (dir.), D'un conte à l'autre, d'une génération à l'autre, Clermont-Ferrand, Presses de l'Université Blaise Pascal, 2008, p. 133-146.

20. Moreux Jean-Pierre, Giros Patricia, Barbe-Bleue, Actes Sud-Papiers, 1989.

21. Castan, Bruno, Coup de bleu, Très Tôt Théâtre 1988, Éditions Théâtrales jeunesse, 2001.

22. Le paratexte dans l'envoi «Merci, Tadeusz » ne laisse guère de doutes sur la référence à Kantor.

23. Rabeux Jean-Michel, La Barbe bleue, L'avant-scène théâtre $n^{\circ}$ 1280, 2010.

24. Dorin Philippe, En attendant le Petit Poucet, L'école des loisirs théâtre, 2001. 
25. Danis Daniel, Bled, L'Arche jeunesse, 2008.

26. Grumberg Jean-Claude, Mange ta main, Heyoka jeunesse Actes Sud-Papiers, 2006.

27. Galea Claudine, Petite poucet, Espaces 34 jeunesse, 2009.

28. Catherine Anne, Ah la la ! quelle histoire, Actes Sud Poche Théâtre, 2008.

29. Granouillet Gilles, Poucet pour les grands, Lansman, 2012.

30. Baratoux Caroline, Le Petit Poucet, Heyoka jeunesse Actes Sud-papiers, 2008.

31. His Eléonore, Le Songe de Baya in La scène aux ados 5, Lansman, 2009.

32. Vers un théâtre contagieux, Répertoire critique du théâtre contemporain pour la jeunesse, volume 2, Editions Théâtrales, Montreuil, 2012, p. 25.

33. On notera que le texte didascalique de la pièce ne donne aucun prénom aux personnages.

34. Serres Michel, Petite Poucette, Editions Le Pommier, 2012.

35. « Le répertoire de théâtre jeunesse entre humour noir et humour multicolore » in Feuerhahn Nelly (édit.), Humoresques, $n^{\circ} 30$, L'enfance du rire, Paris, Editions de la maison des sciences de l'homme, 2010, p. 119-133.

36. On notera cependant qu'il s'agit d'une danse solitaire, côte à côte et non ensemble, comme une annonce de ce que la narratrice raconte de leur séparation future.

37. Pommerat Joël, Cendrillon, op. cit., p. 7.

38. Pillot René, Cendres... Cendrillon, La Fontaine, 1993.

39. La scène aux ados $n^{\circ} 8$, Lansman, 2011.

40. Castan Bruno, La Fille aux oiseaux, Très Tôt Théâtre 1988, Lansman «Les cahiers du soleil debout " 2003, Éditions Théâtrales jeunesse, 2011.

41. Voir à ce sujet l'entretien qu'il a donné à Christian Longchamp pour le Théâtre de la Monnaie à Bruxelles, présenté sur le site Théâtre contemporain, URL: http://www.theatrecontemporain.net/spectacles/Cendrillon/ensavoirplus/idcontent/24079

42. Vers un théâtre contagieux, op. cit., p. 98.

43. Cette expression constitue la matière, ironique, du titre de la pièce de Philippe Dorin, Ils se marièrent et eurent beaucoup, L'école des loisirs théâtre, 2005.

44. Ubu, Scènes d'Europe $\mathrm{n}^{\circ}$ 37/38, avril 2006, p. 65.

45. Sarrazac Jean-Pierre (sous la dir. de), Lexique du drame moderne et contemporain, Belval, Circé, 2004, p. 42 et 43.

46. Se reporter à l'analyse dramaturgique de la pièce dans Vers un théâtre contagieux, op. cit., p. 214-216.

47. Grumberg Jean-Claude, Le Petit Chaperon Uf, Heyoka jeunesse Actes Sud-Papiers, 2005, p. 3940.

48. Voir mon article «Le répertoire de théâtre jeunesse entre humour noir et humour multicolore » in Feuerhahn Nelly (édit.), Humoresques, $n^{\circ} 30$, L'enfance du rire, Paris, Editions de la maison des sciences de l'homme, 2010, p. 119-13.

49. Losco-Lena Mireille, "Rien n'est plus drôle que le malheur". Du comique et de la douleur dans les écritures dramatiques contemporaines, Rennes, Presses universitaires de Rennes, 2011.

50. Pommerat Joël, Le Petit Chaperon rouge, op.cit., p. 17.

51. Ibidem, p. 44.

52. Bernanoce Marie, Vers un théâtre contagieux, op. cit., p. 531.

53. Jouanneau Joël, Le Pavec Marie-Claire, Mamie Ouate en Paoâsie, Actes Sud-Papiers, 1989, p. 6.

54. Ibidem, p. 18.

55. Ibidem, p. 24.

56. Fosse Jon, Le Manuscrit des chiens III, Traduction de Terje Sinding, L'Arche théâtre jeunesse, 2002 ; Le Manuscrit des chiens I, L'arche jeunesse, 2010 ; Le Manuscrit des chiens II, L'arche jeunesse, 2010.

57. Le terme est emprunté à Jean-Pierre Sarrarac, dans La Parabole ou l'enfance du théâtre, Belval, Circé, 2002. 
58. Sarrazac Jean-Pierre, Poétique du drame moderne, Paris, Les Éditions du Seuil, 2012.

59. Nous soulignons. URL: http://www.theatre-contemporain.net/spectacles/Cendrillon/ ensavoirplus/idcontent/24079

60. Pommerat Joël, Gayot Joëlle, Joël Pommerat, Troubles, Arles, Actes Sud, 2009, p. 68.

61. «Je n'écris pas des pièces, j'écris des spectacles ", Ibid., p. 19.

62. Pommerat Joël, Pinocchio, op. cit., p. 7.

63. Entretien avec L. Carré, mai 2013. URL: http://blog.crdp-versailles.fr/ optiontheatremontgeron/index.php/post/29/05/2013/Au-programme-en-TL-1-ann\%C3\%A9eprochaine-\%3A-Cendrillon,-de-Jo\%C3\%ABl-Pommerat

64. Rebotier Jacques, Les Trois Jours de la queue du dragon, Illustrations de Virginie Rochetti, Actes SudPapiers Heyoka jeunesse, 2000.

65. Voir mon article, «La question des genres dans l'enseignement du théâtre contemporain : courants esthétiques et modèles didactiques, des convergences ", in Bernanoce Marie, BrillantAnnequin Anick (sous la dir. de), Enseigner le théâtre contemporain, Grenoble, SCEREN-CRDP de Grenoble, 2009, p. 35-51.

66. Pommerat Joël, Cendrillon, op. cit., p. 5.

67. Ibid., p. 7.

68. Le mot merveilleux est entendu ici au sens que Todorov donne à ce mot dans Introduction à la littérature fantastique, Paris, Seuil, 1970.

69. Pommerat Joël, Cendrillon, op. cit., p. 8-9.

70. Ibid., p. 121.

71. Ibid., p. 120 et 122.

INDEX

Mots-clés : Pommerat (Joël), Cendrillon, conte 